\section{SOBRE LA DEFORMACION EN PERSPECTIVA LINEAL}

\section{Claudi Alsina Catalá}

E. T. S. Arquitectura de Barcelona

Univ. Politécnica de Barcelona

José Antonio Franco Taboada

E. T. S. Arquitectura de La Coruña

Univ. Santiago de Compostela

090-1

\section{Introducción}

En los diferentes sistemas gráficos de representación aparecen siempre una serie de deformaciones relativas entre las imágenes representadas. Dichas deformaciones pueden tener origen diverso:

a) Características de los objetos representados: curvatura, convexidad, opacidad, luz...

b) Limitaciones del observador: agudeza visual, sensibilidad cromática, técnica...

c) Problemas de posiciones relativas entre objetos, observador y marco de representación: alejamiento, oblicuidad..:

además de otros factores que, aún siendo más abstractos, pueden ser determinantes en la concreción de la representación (factores culturales, históricos, estéticos, sociológicos, etc.).

En el caso de los sistemas gráficos utilizados en Arquitectura, cabe conjugar adecuadamente el problema de las deformaciones gráficas con las necesidades de exactitud: en Arquitectura la representación gráfica es a la vez lenguaje de representación y lenguaje de propuesta.

En el presente artículo citamos en el apartado 1 las características fisiológicas oculares que inciden en el problema de la representación. En el apartado 2 recopilamos una serie de referencias históricas. En el apartado 3 se analiza el estudio de las deformaciones en perspectiva lineal en la época actual, para en el apartado 4 estudiar una serie de parámetros que acotan y cuantifican dichas deformaciones, resultando que dichas medidas de deformación son geométricamente deducibles en contraposición a determinados parámetros clásicos, cuya validez residía en resultados empíricos.

\section{El mecanismo de la visión}

Hace casi dos milenios y medio, Platón se atrevía a exponer una teoría de la visión en uno de sus diálogos: "Es una llama que se escapa de cada uno de los cuerpos y que conlleva porciones proporcionadas a la vista, de tal manera que produzca la impresión...». "Las partículas que, provenientes de otros cuerpos, son lanzadas sobre el órgano de la vista son unas más pequeñas y otras mayores que las partes de ese órgano; hay, finalmente, otras que son de la misma dimensión...» (1).

Hoy día, pese a los grandes progresos alcanzados en el conocimiento del mecanismo de la visión, podemos describir cómo se produce la información visual, pero aún no sabemos exactamente cómo ésta se transmite al cerebro y se traduce en la sensación de «ven».

El ojo humano tiene la forma de una esfera, cuyo diámetro oscila entre $24 \mathrm{~mm}$ y $25 \mathrm{~mm}$ (figura 1). Está compuesto de tres capas: la exterior (membrana densa esclerótica), una capa intermedia (coroides) y una capa interna (retina), en la que se distribuyen los receptores de la visión, que pueden considerarse como los terminales del nervio óptico que une el ojo con el cerebro.

Los rayos luminosos que procedentes de un objeto llegan al ojo, inciden sobre la córnea, que los desvía hacia el iris. Este regula la mayor o menor cantidad de luz que debe entrar en el interior del ojo. A través del cristalino se proyectan los rayos sobre la retina, en la cual se forman las imágenes invertidas de los objetos. A fin de que esta imagen aparezca nítida, los cuerpos ciliares modifican la curvatura del cristalino (acomodación) a fin de que objetos situados a diferentes distancias puedan ser enfocados sucesivamente. Las terminaciones nerviosas de la retina

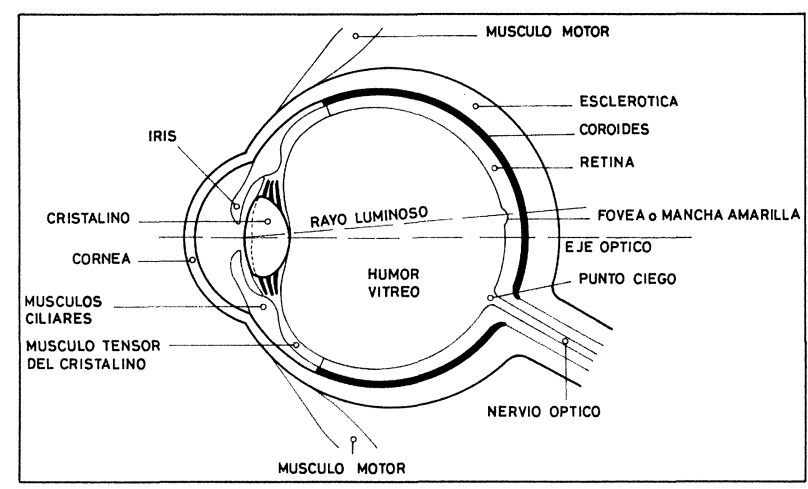

Fig. 1.-Corte esquemático del ojo. 


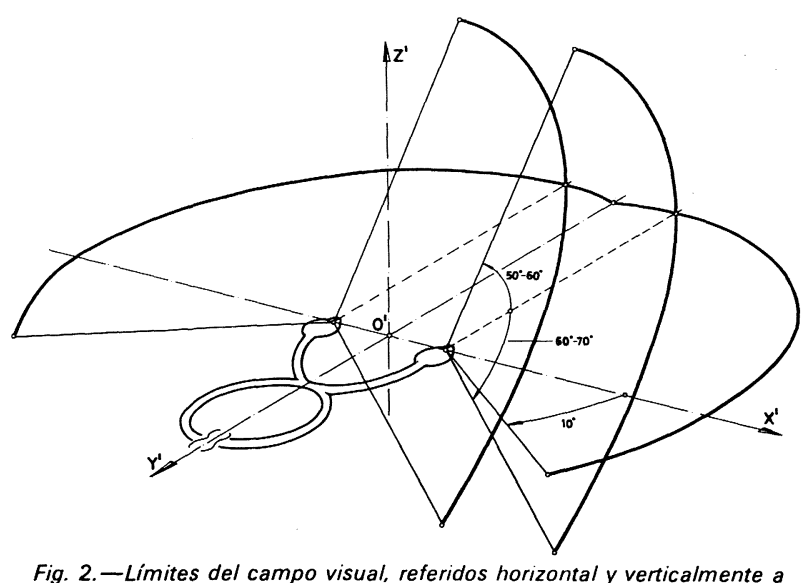

Fig. 2. - Limites del campo visual, referidos horizontal y verticalmente a un sistema de coordenadas cartesianas en el espacio.

convierten la energía luminosa en señales que el nervio óptico transmite al cerebro y éste convierte en la sensación que denominamos «visión».

Los receptores están distribuidos sobre la retina del ojo de una manera no uniforme, apareciendo su mayor concentración sobre la fóvea, o «mácula lútea» (mancha amarilla) del ojo, situada aproximadamente a $5^{\circ}$ del eje óptico (ver figura 1 ), creciendo a partir de la fóvea la separación entre los receptores de una manera concéntrica, disminuyendo además la finura de su estructura y su conexión con los terminales del nervio óptico, en una proporción de uno en la fóvea a cien en los extremos de la retina, lo que influye en la calidad de la señal transmitida al cerebro.

Por otro lado, los receptores son de diferente calidad, siendo algunos de ellos (bastones) únicamente sensibles al grado de claridad, mientras que otros (conos) son sensibles a la longitud de onda y por tanto a los colores, unos sensibles a las longitudes de onda largas (sensación de rojo), otros sensibles a las medias (sensación de verde), y los terceros sensibles a las cortas (sensación de azul). Según las longitudes de onda o colores de los rayos luminosos incidentes'sobre la retina, son activados unos $u$ otros conos, pudiéndose llegar a distinguir una extensísima gama de colores (2). La fóvea está recubierta sólamente de conos muy pequeños y próximos entre sí, estando en el resto de la retina mezclados conos y bastones, aumentando el número relativo de éstos últimos a medida que aumenta la separación de la fóvea, habiendo en el borde de la retina únicamente bastones y siendo por tanto nula en dicha zona la visión de los colores.

En consecuencia, la mayor agudeza visual se da en el centro de la retina, entendiendo por tal la fóvea. Cuando queremos observar con precisión un detalle de un objeto, el ojo se mueve de manera que el cristalino proyecte los correspondientes rayos luminosos sobre la fóvea. El ángulo que puede abarcar la fóvea es únicamente de medio grado aproximadamente, aunque el ángulo que puede considerarse de visión aceptable para pequeños detalles es de $2^{\circ}$ a $3^{\circ}$.
Al observar un cuadro o dibujo cualquiera, los ojos examinan con el mínimo ángulo visual, antes indicado, todos aquellos detalles que atraen nuestra atención (siguiendo un barrido del que apenas somos conscientes). Los detalles pierden su significado fuera del contexto general del dibujo. EI ángulo correspondiente, que incluiría los límites del cuadro o dibujo, ha sido establecido por $\mathrm{H}$. Maertens en unos $27^{\circ}$, correspondientes a una distancia doble de la dimensión más larga del cuadro (3). Lo dicho para un cuadro o dibujo, puede generalizarse para cualquier otro objeto. Además de este ángulo visual de $27^{\circ}$ (que permite apreciar aceptablemente un cuadro en conjunto, sin perder sus detalles) hay el continuo rastreo de los ojos por la superficie observada.

Existe un campo visual general en el cual el ojo (supuesto fijo en la cabeza también fija) puede apreciar los objetos, mediante una visión menos exacta que se denomina periférica que permite captar tanto movimientos laterales, como incluso hacia atrás. Este campo visual es, por tanto, muy amplio, abarcando los siguientes ángulos (figura 2):

- Verticalmente, hasta el límite superior: $50^{\circ}-60^{\circ}$.

- Verticalmente, hasta el límite inferior: $60^{\circ}-70^{\circ}$.

- Horizontalmente, en dirección a la nariz: $60^{\circ}$.

- Horizontalmente, en dirección a la sién: $100^{\circ}$.

Por tanto, el campo visual varía verticalmente de unos $120^{\circ}$ y horizontalmente en unos $200^{\circ}$ (4).

La imagen que se forma sobre la superficie de la retina es una imagen esférica que abarca un amplísimo campo visual (figura 3). La imagen retínica tendría una línea teórica de enfoque indicada en la imagen a trazos y que sólo coincidiría con la imagen en la pequeña zona alrededor de la fóvea, haciéndose el enfoque crítico (y por tanto la imagen lo más nítida posible) únicamente en la fóvea. Por tanto, y tal como se indica en la parte b) de la figura, si una persona quiere apreciar con nitidez otra parte
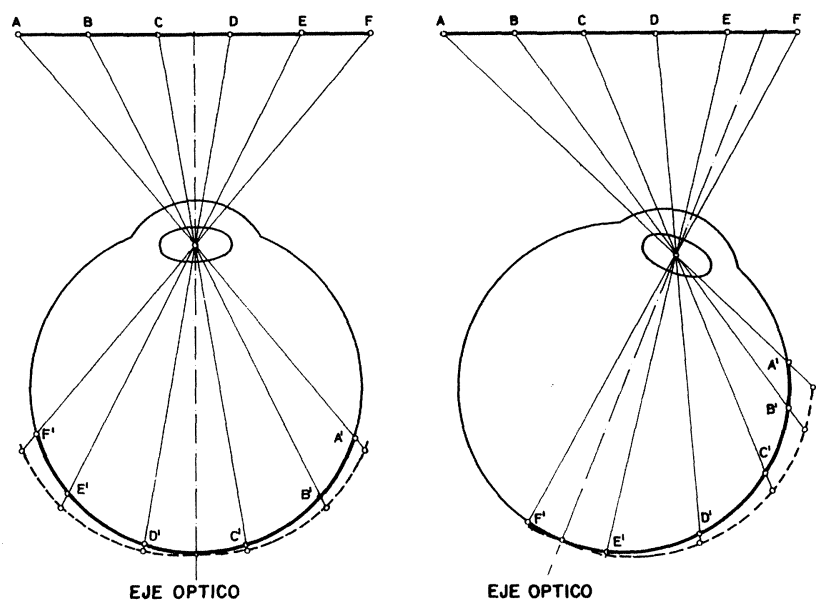

EJE OPTICO

Fig. 3. - Sección esquemática horizontal del ojo, indicando la formación de la imagen $A^{\prime} B^{\prime} C^{\prime} D^{\prime} E^{\prime} F^{\prime}$ de un objeto $A B C D E$. La línea de trazos corresponde con la teórica de enfoque del objeto. 
del objeto, deberá mover el ojo hasta enfocar esa parte sobre la fóvea. La razón del desenfoque gradualmente mayor alrededor de la fóvea, parece deberse, precisamente, a la necesidad de fijar la atención sobre un punto del objeto determinado, sin posibilidad de distracción por los restantes objetos del campo visual general, constituyentes de la visión periférica.

\section{El problema de la representación. Antecedentes históricos}

Con el Renacimiento, y en concreto con León Battista Alberti, la perspectiva se convirtió en el fundamento teórico de la pintura. A Alberti se debe el concepto básico de "perspectiva de un cuerpo", como interacción del plano del cuadro con el cono de rayos visuales (superficie cónica o piramidal), así como el concepto de "línea de igual iluminación», base de la teoría de sombras (5). La pintura adquiría por primera vez un fundamento científico. Alberti comenzó su libro I de "Los tres libros de la pintura» (6) con las siguientes palabras: «Habiendo de escribir acerca de la pintura en estos breves comentarios, tomaré de los matemáticos, para hacerme entender con más claridad, todo aquello que conduzca a mi asunto". Leonardo da Vinci en su "Tratado de la Pintura» (7), sentencia que "La perspectiva es brida y timón de la pintura» e insiste en la conceptualización albertiniana, diciendo que utodos los objetos visibles llegan al ojo por pirámides lineales, cuyo vértice se agota y da fin en el centro de la pupilan. Leonardo compara por primera vez el ojo humano con una cámara obscura, a través de uun experimento que muestra cómo los objetos transmiten imágenes o simulacros que se intersecan en el humor cristalino. Esto queda demostrado cuando por un pequeño orificio circular penetran, en una habitación muy obscura, imágenes de objetos muy iluminados. Si tú recibes imágenes en un papel blanco situado dentro de tal habitación y muy cerca del tal orificio, verás en el papel esos objetos con sus cabales formas y colores, aunque, por culpa de la intersección, a menos tamaño y cabeza abajo...». "Así ocurre dentro de la pupila». Y de acuerdo con el. significado latino de la palabra perspectiva (8) añade que «la perspectiva no es otra cosa que ver un lugar a través de un vidrio plano y perfectamente dibujados todos los cuerpos que están del otro lado del cristal»y.

Ahora bien, y con esto entramos por primera vez en el análisis de la deformación en perspectiva, Leonardo distingue entre perspectiva natural y perspectiva artificial, llamando perspectiva compuesta a la mezcla de ambas, aunque señalando que es preciso "huir de la perspectiva compuesta y atenerse a la simple; no quiere ver el plano en excorzo, sino, hasta donde sea posible, en su forma propia». Para Leonardo, «en perspectiva natural entre cosas de igual magnitud, la más remota parece la menon;; lo que no siempre ocurre con la perspectiva artificial.

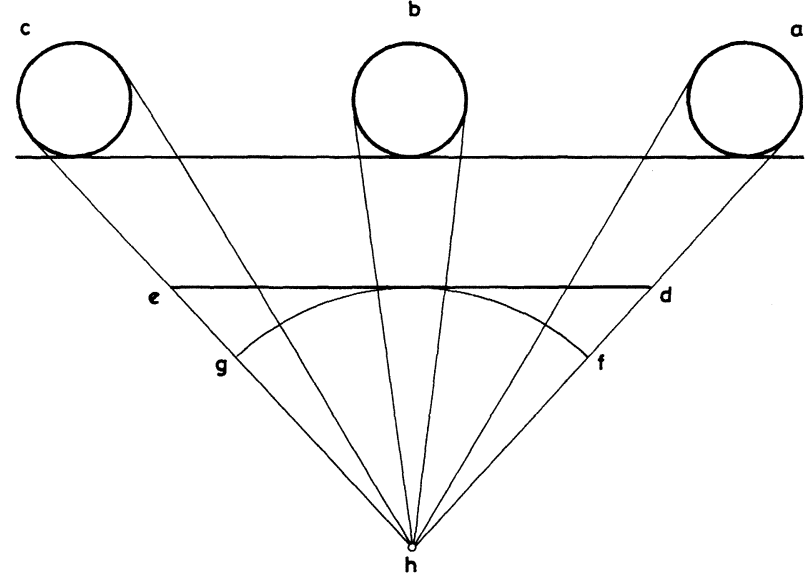

Fig. 4. - "Diagrama" de deformación en el "Tratado de pintura", de Leonardo da Vinci.

Panofsky (9) hace notar la contradicción renacentista entre la teoría de la perspectiva plana y la óptica heredada de la antigüedad, básicamente contraria, que mantenía que las dimensiones visuales, consideradas como proyecciones de las cosas sobre la esfera ocular, no están determinadas por la distancia existente entre los objetos y el ojo, sino exclusivamente por los correspondientes ángulos visuales.

El diagrama que dibuja Leonardo (véase figura 4), plantea gráficamente el problema de la deformación visual. Según Leonardo, usea $d e$ la tal pared, en la cual se representan tres círculos iguales, que están situados tras $d e$, a saber: los círculos $a, b, c$. Puedes comprobar cómo el ojo $h$ ve sobre el plano vertical las secciones de las imágenes: mayores las que están a mayor distancia, menores las que están a menor distancia». Según Leonardo, dicho principio es contrario a la perspectiva natural, término con el que aparentemente Leonardo designa una transcripción «sui generis» de la óptica clásica sobre el plano del cuadro. En cualquier caso, Leonardo no resuelve el problema de la deformación, aunque lo deja claramente planteado. Resulta inconcebible que durante siglos no se haya prestado mayor atención teórica al problema, existiendo aún hoy en día la convicción «populan» de que la perspectiva lineal y su equivalente mecánico, la fotografía, son fieles representaciones de la realidad. Piero della Francesca en su obra «De perspectiva pingendis, plantea también el problema de la deformación de una columnata vista paralelamente al plano del cuadro, pero se limita a demostrar la construcción perspectiva.

Tanto Leonardo como Piero, y de acuerdo con los conocimientos de la época, desconocían el proceso real de la formación de las imágenes en el ojo y lo describían incorrectamente. En general, y empezando por el propio Leonardo, los artistas eludían el problema, siendo famoso el caso de cuadros como "La Escuela de Atenas» de Rafael, en el que las dos esferas situadas en uno de los lados del cuadro están representadas como círculos, cuando sus perspectivas lineales deberían ser claramente elipses. 
Es preciso llegar al siglo XVII y a una obra fundamental en el desarrollo de la cultura occidental, el «Discurso del Método» de René Descartes, para aclarar la forma real de la imagen retínica, en un experimento realizado por el propio Descartes y que describe en el Discurso quinto de la Dióptrica (10), consistente en tomar uel ojo de un hombre muerto recientemente o, en su defecto, el de un buey o el de otro animal» y colocándolo debidamente preparado y recubierto de un material translúcido, como una cáscara de huevo, en el orificio de una ventana expresamente construida en una habitación totalmente cerrada, o cámara obscura, «veréis un dibujo, no sin admiración ni placer, que presentará muy simplemente en perspectiva todos los objetos situados fuera» e iluminados por el sol. $Y$ aunque Descartes plantea la forma esférica de la imagen resultante sobre la retina, no puede evitar afirmar que «las imágenes, cuya aparición provocamos sobre una tela blanca y en el interior de una cámara obscura, se forman de igual modo y por idéntica razón que se forman en la parte interior del ojon. Desde un punto de vista geométrico, deja en el aire la antigua confusión del ilusionismo que la perspectiva lineal en la pintura había introducido en el Renacimiento.

\section{El estudio de las deformaciones en la época actual}

Al llegar al siglo XIX parecía que el problema podía ser resuelto definitivamente. Pero, como llega a decir Panofsky (11), se trata de un «enmarañado problema», o como irónicamente manifiesta $\mathrm{E}$. H. Gombrich (12), «si puede servir de consuelo al lector, permítame afirmar mi convicción de que muchos tratadistas de perspectiva se han armado un lío en este punto, sin excluirme a mí mismo, desde luegor.

El primero en estudiar modernamente los aspectos filosóficos y psicológicos de la perspectiva lineal fue el alemán Guido Hauck, uno de los máximos exponentes de la Geometría Descriptiva (13). Pero quien planteó de un modo sistemático una interpretación psicológica, dentro ciel campo de la evolución de la pintura, fue Erwin Panofsky, en la obra ya citada "La perspectiva como forma simbólica», publicada en 1927, y cuyo contenido desarrolló más ampliamente en una conferencia pronunciada en un curso de verano organizado en 1952 por la Universidad de Upsala y recogido en la obra publicada en 1962 con el título de "Renacimiento y renacimientos en el arte occidental» (14). En esta última obra, Panofsky concluye que "dicho en pocas palabras, el espacio de la pintura helenística y romana carece de las dos cualidades que caracterizan al espacio que el arte "moderno" presupone y presenta hasta Picasso: continuidad ( $y$, por tanto, mensurabilidad) e infinitud», este espacio... «en la práctica representacional... fue presupuesto y ejemplificado por lo que conocemos con el nombre de perspectiva geométrica exacta». Después de anali- zar el concepto de la perspectiva como transformación proyectiva, Panofsky plantea el problema básico de la deformación como consecuencia del Teorema Octavo de la Optica de Euclides, que afirma que «la diferencia aparente entre magnitudes iguales vistas desde distancias desiguales, no es en absoluto proporcional a esas distancias», en patente desacuerdo con las reglas de la perspectiva de Brunelleschi y Alberti; hasta el punto de que los traductores renacentistas de la Optica euclidiana, corrigieron o suprimieron el Octavo Teorema. La razón de la contradicción estriba en la imposibilidad de desarrollar sobre el plano del cuadro de la perspectiva la imagen esférica sobre la retina, como se conocía ya de antiguo en cartografía.

M. H. Pirenne vuelve sobre el problema afirmando «que no siempre encuentro fácil entender lo que el profesor Panofsky, y otros historiadores de arte influidos por su tesis, quieren decir exactamente desde una posición óptica, fisiológica y psicológica» (15). Pirenne ha realizado fotografías con cámaras de orificio puntual, a fin de eliminar las distorsiones producidas por los objetivos, así como perspectivas mediante ordenadores en la Universidad de Oxford (16). Curiosamente, Pirenne comete la equivocación evidente de afirmar que La Gournerie, al reemplazar las figuras de las dos esferas de $\mathrm{La}$ Escuela de Atenas, de Rafael, por sus perspectivas elípticas correctas, «se encontró con que estas correctas proyecciones centrales, que producían las mismas imágenes retínicas que hubieran producido las esferas reales, eran inaceptables», confundiendo la imagen plana con la proyectada sobre la superficie cóncava de la retina, que el propio autor analiza detalladamente al principio de su obra. Pese a estudiar con detalle las deformaciones en perspectiva lineal, no consigue establecer una explicación coherente que logre superar la de Panofsky porque pese a fotografías y ordenadores, establece erróneamente «el punto de vista» en relación con «el plano del cuadro», ignorando la importancia del rayo principal como referencia de la deformación en relación con el eje óptico del ojo.

Finalmente, una última obra importante que intenta resolver el problema es la de E. H. Gombrich, "Arte

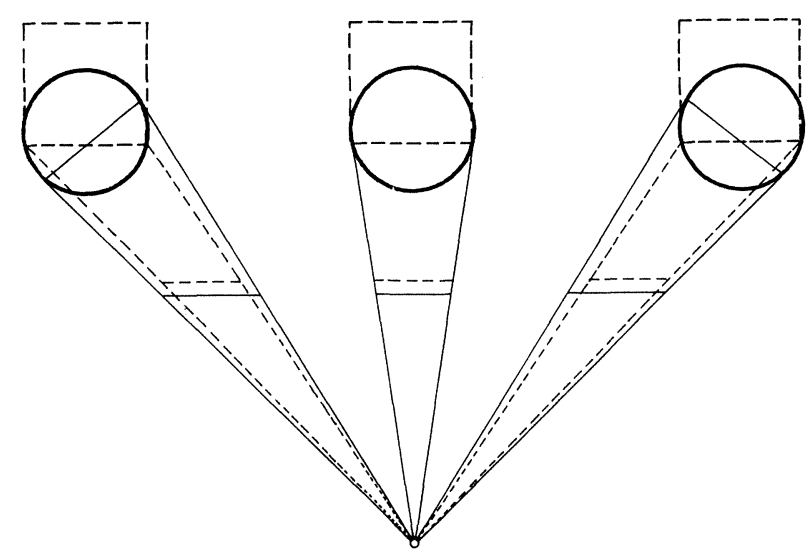

Fig. 5.-Dibujo de B. A. R. Carter, en "Arte e llusión", de E. H. Gombrich. 
Informes de la Construcción/351-352

e ilusión. Estudio sobre la psicología de la representación pictórical, que riza el rizo de las interpretaciones y contrainterpretaciones que arrancan del famoso dibujo de Leonardo de la figura 4, en el que se plantea el resultado paradógico, tambiéń señalado ya por Piero della Francesca, sobre las columnas laterales. Gombrich recoge un dibujo de B. A. R. Carter (véase figura 5) y explica que ula razón de la paradoja no es que las leyes de la perspectiva sean inexactas, sino que a veces sus resultados ordinarios de la proyección geométrica nos pillan por sorpresa. Las columnas, desde luego, se extienden en anchura y en profundidad, y la extensión que se aleja del plano frontal de la elevación, es lo que causa la ligera anomalía. La cuestión se aclara si imaginamos pilastras cuadradas en vez de columnas, y todavía más si imaginamos dichas pilastras pintadas de rojo en el frente y de verde en los lados. La perspectiva demuestra que en tal caso los idénticos frentes rojos de las pilastras aparecerán como idénticos rectángulos rojos en el plano proyectivo, pero mientras que el pilar que se nos enfrenta directamente no mostrará nada de sus lados verdes, veremos una cantidad creciente de verde a medida que se ve una parte mayor de los lados de las pilastras. Con un ojo, como muestra el diagrama, nunca vemos todo el ancho de una columna, ya que las tangentes formadas por las rectas de la visión tocan a la circunferencia tanto más cerca una de otra, cuanto más cerca estamos. A muy corta distancia, este ligero inesperado aumento del área captada por el ojo al alejarnos, compensa en parte la disminución de tamaño debida a la mayor distancial.

En realidad se trata de un sofisma geométrico, puesto que observando atentamente el dibujo de B. A. R. Carter que presenta Gombrich como demostración, se observa que las imágenes sobre el teórico plano del cuadro de las columnas más alejadas son mayores que la de la más cercana, al contrario de lo que nos dice la experiencia. El propio Gombrich añade a continuación de su aparente demostración que utodo eso, sin duda, es algo desconcertante».

\section{Cuantificaciones matemáticas de la deformación}

El estudio matemático de las deformaciones en perspectiva lineal se ha planteado con anterioridad a un nivel elemental, puramente empírico, partiendo de aproximaciones gráficas (17).En la mayoría de manuales aparece el esquema representado en la figura 6, como resultado de admitir apriorísticamente la cuantificación de la deformación vía la expresión $D=\operatorname{tag}^{2} \alpha / 2$, siendo $\alpha$ el ángulo visual, es decir, $\alpha / 2$ es la desviación angular respecto del rayo principal ortogonal al plano del cuadro. Empíricamente se ha verificado que $D$ aporta un cierto grado de información» pero, en general, no existe base teórica que aclare el «tipo» de información aportada. En esta sección introduciremos una definición genérica de deformación, deduciremos teó-

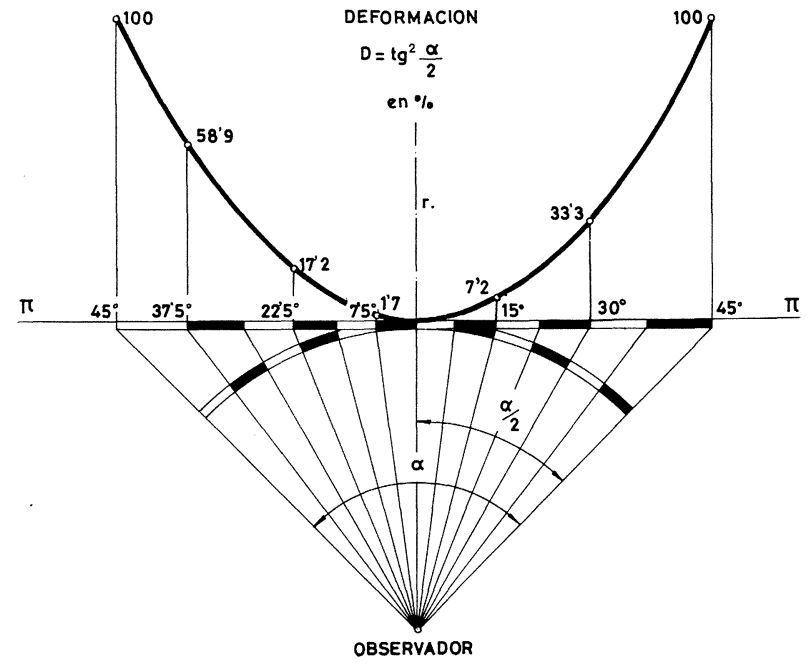

Fig. 6. -Diagrama de deformación simplificado.

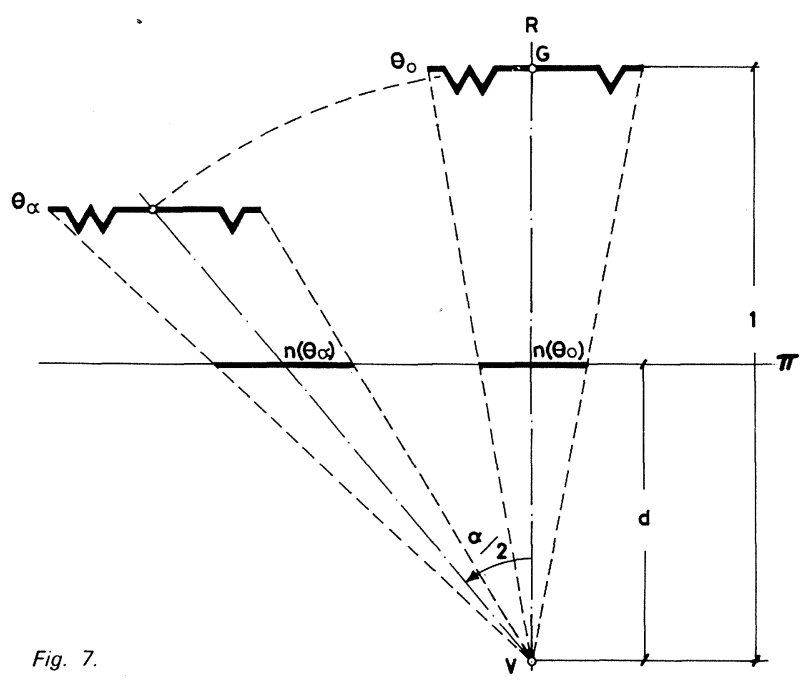

ricamente $D$ y propondremos una nueva medida alternativa que, a nuestro entender, se ajusta más a la idea de deformación en perspectiva lineal.

Para ello empecemos introduciendo una serie de notaciones:

V: Punto de vista del observador.

$\pi$ : Plano del cuadro.

$\mathrm{R}$ : Rayo principal o recta ortogonal de $\mathrm{V}$ a $\pi$.

d: Distancia de $\vee$ a $\pi$.

$\sigma_{\sigma}$ : Objeto situado a distancia unidad del observador con centro de gravedad $G$ sobre el rayo principal $R$.

$\alpha / 2$ : Desviación angular.

$\sigma_{\alpha}$ : Objeto que resulta al desplazarse paralelamente al plano del cuadro $\pi$ el objeto $\sigma_{\text {o }}$ habiendo girado su centro de gravedad $\alpha / 2$ grados respecto del rayo principal.

$\pi\left(\sigma_{\alpha}\right)$ : Figura en $\pi$ que representa a $\sigma_{\alpha}$.

En el esquema 7 sintetizamos gráficamente las notaciones introducidas. 
Nótese que el objeto referencial $\sigma_{\text {o }}$ puede ser tanto plano como tridimensional pero la figura $r\left(\sigma_{0}\right)$ del plano del cuadro siempre es plana. Es también fundamental notar que al pasar de $\sigma_{\mathrm{o}}$ a $\sigma_{\alpha}$ el centro de gravedad $\mathrm{G}$ habrá girado $\alpha / 2$ grados, y por tanto la distancia unidad de $\mathrm{V}$ a $\mathrm{G}$ se mantiene, mientras que $\sigma_{\alpha}$ se mantiene paralelo a $\sigma_{\circ}$ y al plano del cuadro, graciaș a lo cual ula forma» de $r\left(\sigma_{d}\right)$ y $r\left(\sigma_{\alpha}\right)$ es la misma.

DEFINICION FUNDAMENTAL: Fijado un objeto

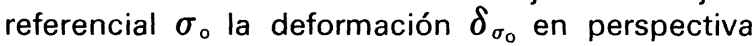
lineal viene dada por:

$$
\delta_{\sigma_{0}}=\frac{\text { Area } r\left(\sigma_{\alpha}\right)-\operatorname{Area} r\left(\sigma_{0}\right)}{\text { Area } r\left(\sigma_{0}\right)}
$$

Evidentemente $\delta_{\sigma_{0}}$ depende de $\sigma_{\circ}$ y de $\alpha$ y $0 \leq \delta_{\sigma_{0}}$. Cuando $\alpha=0\left(\sigma_{\mathrm{o}}\right.$ no se ha movido o el observador está en el infinito) resulta $\delta_{\sigma_{0}}=0$. Los casos de interés son aquellos en que $0<\delta_{\sigma_{0}}$, es decir, la deformación no es nula.

Vamos a obtener ahora expresiones explícitas de $\delta_{\sigma_{\circ}}$ en el caso de dos tipos diferentes de objetos referenciales: cuadrados y esferas.

\section{Deformación con objeto referencial: un cuadrado}

Si $\sigma_{0}$ es un cuadrado $C_{0}$ de lado $L$ tanto $r\left(\sigma_{0}\right)$ como $r\left(\sigma_{\alpha}\right.$ serán también cuadrados. De acuerdo con la figura 8 , tendremos a la vista de la semejanza entre los triángulos $\widehat{V A}_{0} C_{0}$ y $\widehat{V A}_{0} C_{0}, \widehat{V A}_{\alpha} M_{\alpha}$ y $\widehat{V A}_{\alpha} M_{\alpha}$ :

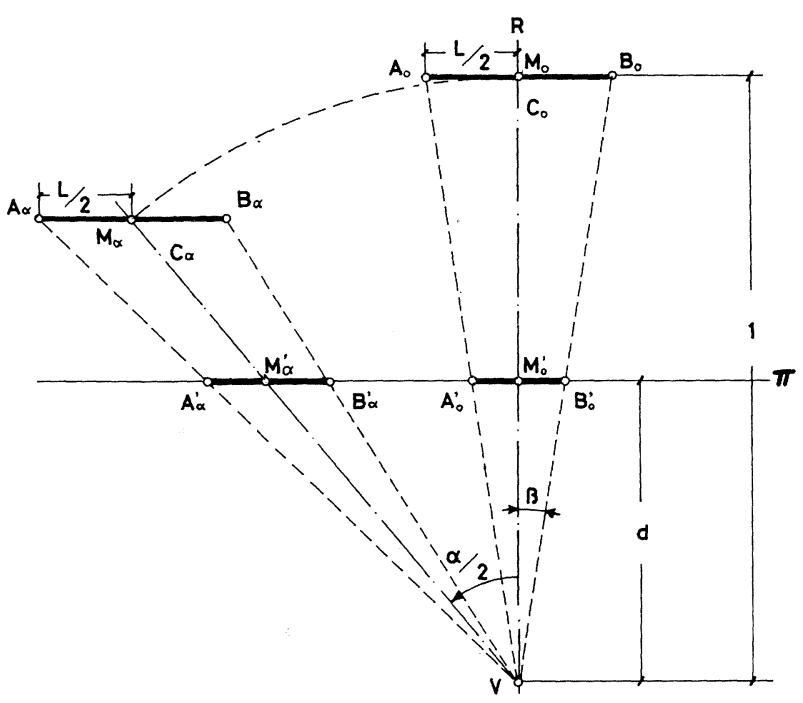

$$
\frac{\overline{\mathrm{A}_{0} \mathrm{M}_{0}}}{{\overline{\mathrm{A}_{0} \mathrm{M}_{0}}}_{0}}=\frac{\overline{\mathrm{VM}_{0}}}{\overline{\mathrm{VM}}_{0}} \text { y } \frac{\overline{\mathrm{A}_{\alpha} \mathrm{M}_{\alpha}}}{{\overline{\mathrm{A}_{\alpha} \mathrm{M}_{\alpha}}}_{\alpha}}=\frac{\overline{\mathrm{VM}_{\alpha}}}{\overline{\mathrm{VM}}_{\alpha}}
$$

de donde se deduce que:

$$
\begin{aligned}
& \frac{\overline{\mathrm{A}_{0} \mathrm{M}_{0}}}{\overline{\mathrm{A}_{\alpha} \mathrm{M}_{\alpha}^{\prime}}}=\frac{\overline{\mathrm{A}_{0} \mathrm{M}_{0}} \cdot \frac{\overline{\mathrm{VM}_{0}}}{\overline{\mathrm{VM}_{0}}}}{\overline{\mathrm{A}_{\alpha} \mathrm{M}_{\alpha}} \cdot \frac{\overline{V M_{\alpha}}}{\overline{\mathrm{VM}_{\alpha}}}}=\frac{\overline{\mathrm{VM}_{\alpha}}}{\overline{M_{\alpha}}}=\cos \alpha / 2, \\
& \text { al ser }{\overline{A_{0} M_{0}}}_{0}={\overline{A_{\alpha} M}}_{\alpha}=L / 2 \text { y } \overline{V M}_{0}=\overline{V M}_{\alpha}=1 \text {. }
\end{aligned}
$$

Por tanto la deformación valdrá:

$$
\delta_{\mathrm{C}_{0}}=\frac{\text { Area } r\left(\mathrm{C}_{\alpha}\right)}{\text { Area } r\left(\mathrm{C}_{0}\right)}-1=\frac{\left(2 \overline{\mathrm{A}_{\alpha} \mathrm{M}_{\alpha}}\right)^{2}}{\left(2 \overline{\mathrm{A}_{0} \mathrm{M}_{0}}\right)^{2}}-1=\frac{1}{\cos ^{2} \frac{\alpha}{2}}-1=\operatorname{tag}^{2} \frac{\alpha}{2}
$$

expresión que coincide (!) con el parámetro clásico de deformación antes comentado.

\section{Deformación con objeto referencial: una esfera}

Siendo la esfera el cuerpo tridimensional de máxima simetría, parece lógico, recuperando la tradición pictórica antes comentada, calcular la deformación $\delta_{E_{0}}$ para el caso de una esfera $E_{0}$ de radio $r$ (figura 9 ). Al considerar el plano $\pi$ vertical, con arreglo a una perspectiva clásica, el plano pasando por los centros de las esferas podría ser horizontal y los «círculos» aparentes de las esferas con respecto al obsenvador (y que éste veía iguales al girar los 


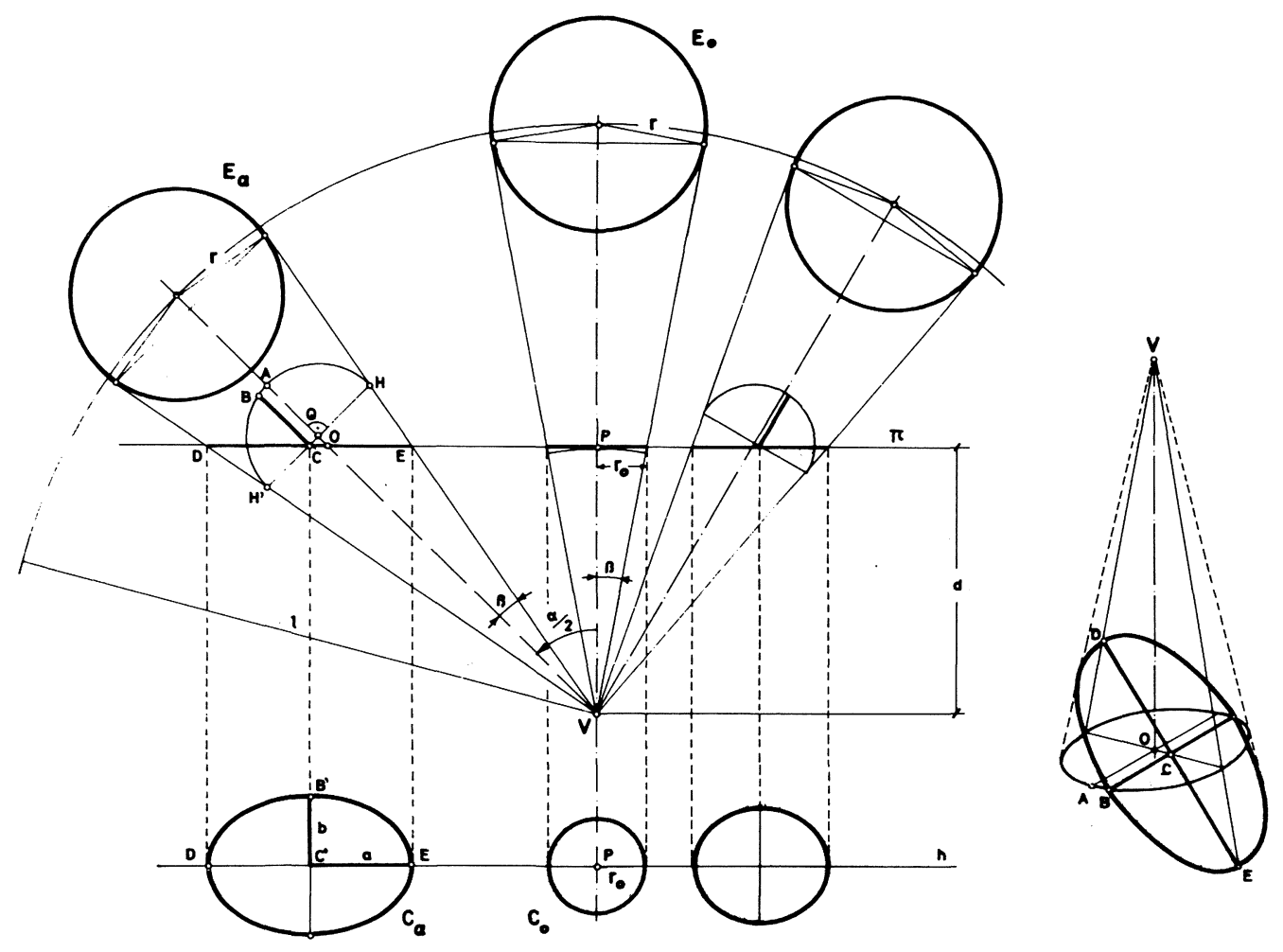

Fig. 9. - Perspectivas de tres esferas iguales y equidistantes del punto de vista $\mathrm{V}$.

ojos hasta observar enfocadamente cada esfera) aparecen claramente deformados como elipses. Siguiendo la figura 9, tendremos en primer lugar que $E_{0}$ se representará en $\pi$ por el círculo $C_{0}$ de radio $r_{0}$ igual a:

$$
\mathrm{r}_{0}=\mathrm{d} \cdot \operatorname{tag} \beta
$$

al $\operatorname{ser} \operatorname{sen} \beta=r / 1$. Por tanto:

$$
\text { Area } r\left(E_{0}\right)=\text { Area }\left(C_{0}\right)=\pi r_{0}^{2}=\pi d^{2} \operatorname{tag}^{2} \beta
$$

Como la esfera girada $E_{\alpha}$ se representa en $\pi$ mediante la elipse $C_{\alpha}$, precisamos calcular los semiejes $a$ y $b$ de dicha elipse. Para determinar el semieje mayor $a$, establecemos las siguientes relaciones trigonométricas:

En el triángulo rectángulo OPV se cumple:

$$
\overline{\mathrm{OV}}=\overline{\mathrm{VP}} / \cos (\alpha / 2)=\mathrm{d} / \cos (\alpha / 2)
$$

Por el teorema del seno aplicado al triángulo $\widehat{\mathrm{OEV}}$; se tiene:

$$
\frac{\overline{\mathrm{OE}}}{\operatorname{sen} \beta}=\frac{\overline{\mathrm{OV}}}{\operatorname{sen}(90+\alpha / 2-\beta)}
$$

de donde por (1) resulta:

$$
\overline{\mathrm{OE}}=\frac{\operatorname{sen} \beta}{\operatorname{sen}(90+\alpha / 2-\beta)} \cdot \overline{\mathrm{OV}}=\frac{\mathrm{d} \operatorname{sen} \beta}{\cos (\alpha / 2-\beta) \cos \alpha / 2}
$$

Análogamente, el teorema del seno aplicado al triángulo $\widehat{\mathrm{DOV}}$ da lugar a:

$$
\overline{\mathrm{DO}}=\frac{\operatorname{sen} \beta}{\operatorname{sen}(90-\alpha / 2-\beta)} \cdot \overline{\mathrm{OV}}=\frac{\mathrm{d} \operatorname{sen} \beta}{\cos (\alpha / 2+\beta) \cos \alpha / 2}
$$


De (2) y (3) se sigue el valor del semieje a:

$$
a=\frac{\overline{\mathrm{OE}}+\overline{\mathrm{DO}}}{2}=\frac{\mathrm{d} \operatorname{sen} \beta}{2 \cos \alpha / 2} \cdot\left(\frac{1}{\cos (\alpha / 2-\beta)}+\frac{1}{\cos (\alpha / 2+\beta)}\right),
$$

y tras las oportunas simplificaciones resulta:

$$
\mathrm{a}=\frac{\mathrm{d} \operatorname{sen} \beta \cos \beta}{\cos (\alpha / 2+\beta) \cos (\alpha / 2-\beta)}
$$

Vamos a calcular ahora el semieje menor $b$. En el triángulo rectángulo $\widehat{\mathrm{COO}}$ se tiene $\widehat{\mathrm{Q}}=90^{\circ}, \widehat{\mathrm{O}}=90-\alpha / 2$ y $\widehat{\mathrm{C}}=\alpha / 2$ y por tanto resulta tras ciertas operaciones:

$$
\overline{\mathrm{CQ}}=\overline{\mathrm{CO}} \cdot \operatorname{sen}(90-\alpha / 2)=(\overline{\mathrm{CE}}-\overline{\mathrm{OE}}) \cos \alpha / 2=\frac{\mathrm{d} \operatorname{sen}^{2} \beta \operatorname{sen} \alpha / 2}{\cos (\alpha / 2-\beta) \cos (\alpha / 2+\beta)}
$$

En el triángulo $\widehat{\mathrm{CHE}}$ es $\hat{\mathrm{H}}=90-\beta, \widehat{\mathrm{C}}=\alpha / 2$ y $\widehat{\mathrm{E}}=90+\beta-\alpha / 2$ y aplicando una vez más el teorema del seno:

$$
\frac{\overline{\mathrm{CH}}}{\operatorname{sen}(90+\beta-\alpha / 2)}=\frac{\overline{\mathrm{CE}}}{\operatorname{sen}(90-\beta)},
$$

de donde al ser $\overline{\mathrm{CH}}=\overline{\mathrm{CQ}}+\overline{\mathrm{QH}}$ y $\overline{\mathrm{CE}}=\mathrm{a}$, se deduce de (6), (4) y (5):

$$
\overline{\mathrm{QH}}=\overline{\mathrm{CH}}-\overline{\mathrm{CQ}}=\frac{\operatorname{sen}(90+\beta-\alpha / 2)}{\operatorname{sen}(90-\beta)} \cdot \overline{\mathrm{CE}}-\overline{\mathrm{CQ}}=\frac{\mathrm{d} \operatorname{sen} \beta \cos \beta \cos \alpha / 2}{\cos (\alpha / 2+\beta) \cos (\alpha / 2-\beta)}
$$

Finalmente al ser $\overline{\mathrm{BC}}$ la altura sobre la hipotenusa $\overline{\mathrm{HH}}^{\prime}$ del triángulo rectángulo $\widehat{\mathrm{BH}} \mathrm{H}^{\prime}$, resulta por el teorema de la altura, el valor del semieje menor $b$ :

$$
b=\overline{B C}=\sqrt{\overline{\mathrm{CH}} \cdot \overline{\mathrm{CH}^{\prime}}}=\sqrt{(\overline{\mathrm{QH}}+\overline{\mathrm{CQ}})(\overline{\mathrm{QH}}-\overline{\mathrm{CQ}})}=\sqrt{\overline{\overline{\mathrm{BH}}^{2}}-\overline{\mathrm{CQ}^{2}}}
$$

de donde por (5) y (7) se obtiene:

$$
b=\frac{d \operatorname{sen} \beta}{\sqrt{\cos (\alpha / 2+\beta) \cos (\alpha / 2-\beta)}}
$$

De (4) y (8) deducimos inmediatamente el área de la elipse $E_{\alpha}$ :

$$
\text { Area } r\left(E_{\alpha}\right)=\text { Area }\left(C_{\alpha}\right)=\pi a \cdot b=\frac{\pi d^{2} \operatorname{sen}^{2} \beta \cos \beta}{[\cos (\alpha / 2+\beta) \cos (\alpha / 2-\beta)]^{3 / 2}}
$$

y por tanto la deformación relativa a dichas elipses será:

$$
\delta_{\mathrm{E}_{0}}=\frac{\operatorname{Area} \mathrm{r}\left(\mathrm{E}_{\alpha}\right)}{\operatorname{Area} \mathrm{r}\left(\mathrm{E}_{0}\right)}-1=\frac{\frac{\pi \mathrm{d}^{2} \operatorname{sen}^{2} \beta \cos \beta}{[\cos (\alpha / 2+\beta) \cos (\alpha / 2-\beta)]^{3 / 2}}}{\pi \mathrm{d}^{2} \operatorname{tag}^{2} \beta}-1
$$

o equivalente:

$$
\delta_{\mathrm{E}_{0}}=\frac{\cos ^{3} \beta}{\left(\cos ^{2} \alpha / 2-\operatorname{sen}^{2} \beta\right)^{3 / 2}}-1
$$


Cuando $\beta$ tiende a 0 la deformación $\delta_{\mathrm{E}_{0}}$ tiende a $\frac{1}{\cos ^{3} \alpha / 2}-1$. Curiosamente para $\alpha$ tendiendo a 0 , resulta que $\delta_{\mathrm{E}_{0}}$ tiende a 0 . También puede reescribirse $\delta_{\mathrm{E}_{0}}$ en términos de la $\varepsilon=\mathrm{b} / \mathrm{a}$ resultando:

$$
\delta_{\mathrm{E}_{0}}=\frac{1-\varepsilon^{3}}{\varepsilon^{3}}
$$

Notemos ahora que $\delta_{\mathrm{E}_{0}}$ depende a la vez de $\alpha$ y de $\beta$, o lo que es lo mismo, de $\alpha$ y de r, pues $\mathrm{r}=\operatorname{tag} \beta$ es el radio de la esfera $E_{0}$. Así, mientras $\delta_{\mathrm{C}_{0}}=\operatorname{tag}^{2} \alpha / 2$ era independiente del tamaño del cuadrado, $\delta_{\mathrm{E}_{0}}$ sí que depende del tamaño de la esfera. Ello no es de extrañar dado que los cuadrados del caso I se representaban en el plano del cuadro por cuadrados, pero las esferas son representadas por elipses cuya excentricidad depende del tamaño de las esferas originales.

\section{Epílogo}

Hasta aquí hemos estudiado las deformaciones en perspectiva lineal, pero podrían derivarse análogas consideraciones respecto de la axonometría (18), cuantificando las deformaciones tal y como hace, en una primera aproximación, Harald Berns (19). De ello podría deducirse enseguida el hecho de que las axonometrías ortogonales y en concreto las dimetrías, dan una aproximación mayor de la realidad que las axonometrías oblícuas. Ello es consecuencia precisamente de que en las axonometrías ortogonales puede considerarse la dirección de proyección perpendicular al plano del cuadro coincidente con el rayo principal, lo que no ocurre en las axonometrías oblícuas.

El gran tema teórico (de evidentes repercusiones prácticas) que se esconde detrás del problema de las deformaciones en representaciones gráficas, es el de llegar a formular científicamente una «teoría general de la información de los sistemas gráficos de representaciones». Las representaciones como los lenguajes, sintetizan unos contenidos sintácticos y unas aportaciones semánticas. En un dibujo o un cuadro existen unos trazos, unos códigos semánticos, unas dosis de simbología, de equilibrio, de ritmo..., toda una extensa gama de matices $y$ conceptos que, en gran parte, pueden ser cuantificados. ¿Qué información transmiten los diferentes sistemas gráficos? ¿Cómo se mide dicha «información»? Quizás el problema esté hoy lejos aún de solución. "Hay que mirar mucho para llegar a ven», diría Paul Claudel, ¿ver qué?

La Coruña, 1982
1. Platón: "Obras Completas» (Timeo o de la naturaleza). Traducción del griego por Francisco P. Samaranch. Ed. Aguilar, pp. 1160 y 1161,1969

2. Véase la obra "Colon» de Frans Gerritsen, que puede ser consultada para mayor detalle en relación con la percepción del color. Ed. Blume, Barcelona, 1976.

3. Maertens, $\mathrm{H} .:$ «Der optische masstab oder die theorie und praxis des ästhetischen sehens in den bildenden künsten, Berlín, 1884». Citado por Rudolf Arnheim en "La forma visual de la Arquitecturan. Ed. Gustavo Gili, Barcelona, 1978

4. Según Frans Gerritsen, M. H. Pirenne (véase bibliografía), da un ángulo horizontal superior, de $104^{\circ}$. Rudolf Arnheim coincide en el ángulo horizontal, pero en el vertical baja a $110^{\circ}$, con unos $45^{\circ}$ sobre el nivel del ojo y $65^{\circ}$ por debajo.
5. Véase «Storia della Geometría descriptiva», de Gino Loria, pp. 6 y 7 , Milán, 1921.

6. Edición facsímil de 1980 del C. O. de Aparejadores y Árquitectos Técnicos de Murcia, de la traducción de Don Diego Antonio Rejón de Silva, «El tratado de la pintura por Leonardo Da Vinci y los tres libros que sobre el mismo arte escribió León Bautista Alberti», en Madrid, en la Imprenta Real, MDCCLXXXIV.

7. Edición preparada por Angel González Gárcía, Editora Nacional, Madrid, 1976

8. Erwin Panofsky comienza su obra "La perspectiva como forma simbólica», diciendo: "Item perspectiva es una palabra latina; significa mirar a través». Así es como Durero trató de circunscribir el concepto de perspectiva. 
9. Obra citada.

10. René Descartes: «Discurso del método, dióptrica, meteoros y geometríal, con prólogo, traducción y notas de Guillermo Quintás Alonso. Eds. Alfaguara, 1981.

11. Enwin Panofsky, obra citada, nota 8.

12. E. H. Gombrich: «Arte e ilusión. Estudio sobre la psicología de la representación pictórica». Gustavo Gili 1979, correspondiente a la quinta edición inglesa de 1977.

13. Guido Hauch publicó los primeros estudios sobre aspectos psicológicos y artísticos de la perspectiva tradicional en obras como: «Die subjecktive und die horizontalen curvaturen des dorischen styls», Stuttgart, 1879, y "Die malerische perspectivel, Berlín, 1882 (citadas por E. H. Gombrich y Enwin Panofsky). Un amplio comentario a sus aportaciones al campo de la geometría descriptiva se encuentra en la obra ya citada de Gino Loria, pp. 278 y siguientes.

14. Erwin Panofsky: «Renacimiento y renacimientos en el arte occidental», publicada por Alianza Editorial, Madrid, 1975 (véase en concreto el capítulo 3 "I primi lumi»: la pintura del trecento italiano y su impacto sobre el resto de Europan, pp. 175 y siguientes. Para conocer la personalidad del autor puede consultarse el prólogo de Enrique
Lafuente Ferrari, destinado a servir de "Introducción a Panofsky» en su "Estudios de Iconología», publicada por Alianza Editorial, Madrid, 1972.

15. M. H. Pirenne: obra citada. Nota al pie de la página 117 y siguientes.

16. Mediante una computadora inglesa KDF 9 y una trazadora Calcomp, la mayor parte del trabajo fue programado en Fortran, según las rutinas básicas expuestas en «A User's guide to the Culham Graphical Output System», de F. M. Larkin.

17. En obras traducidas al castellano por Georg Schaanwächter en «Perspectiva para arquitectos», Ed. Gustavo Gili, BarceIona, 1970, pág. 27 y en dos obras de Reiner Thomae «Perspectiva y Axonometría» y «El encuadre en la perspectivall de la misma editorial y en la que presenta resultados contradictorios.

18. También se podría establecer un paralelismo análogo al de Bruno Reichlin, en su artículo "L'assonometria come progetto" publicado en el n. ${ }^{\circ} 22$ de Lotus (1979), y en relación con "La perspectiva como forma simbólica», de Panofsky.

19. En su obra «Sistemas de representación gráfica», Ediciones Urmo, 1969.

\section{publicaciones del i.e.t.c.c.}

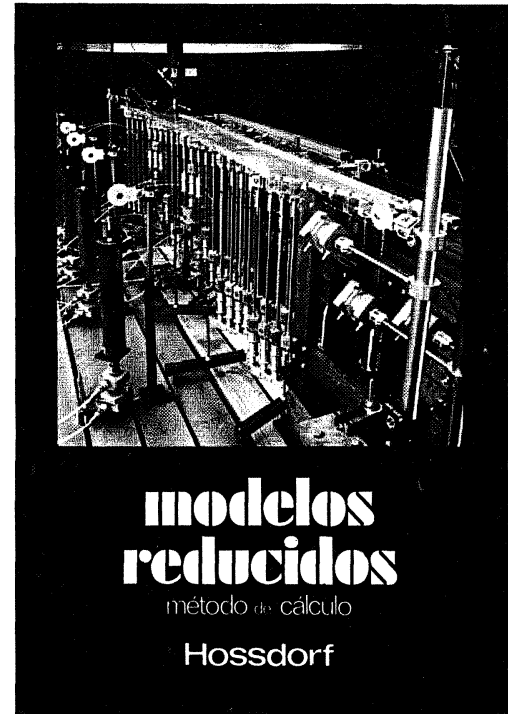

Modelos reducidos. Método de cálculo

H. Hossdorf, Ingeniero Civil

La técnica de los ensayos en modelos reducidos de estructuras sufre hoy dia una decisiva metamorfosis. Hasta hace poco era un medio mas bien de artesania, que no siempre era tomado en serio por los académicos teorizantes para comprender el comportamiento resistente de las estructuras complejas y al que se acudió las más de las veces, como a un último remedio debido a sus indiscutibles insuficien cias. Sin embargo, en poco tiempo y gracias a su conexión con los ordenadores digitales, se ha trans formado en un instrumento cientificamente valioso, que no puede quedar a un lado en la práctica
diaria del Ingeniero Proyectista.

Un volumen encuadernado en cartoné plastificado con lomo de tela, de $17 \times 24 \mathrm{~cm}$, compuesto 250 páginas, 158 figuras $y$ fotografias.

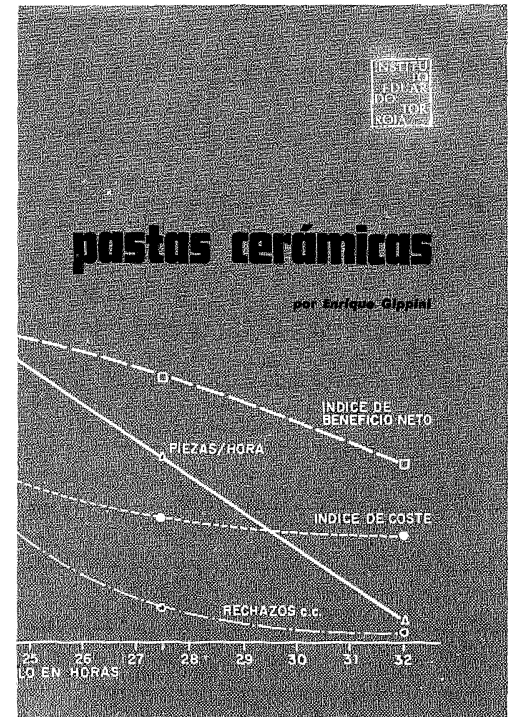

PASTAS CERAMICAS

\section{Enrique Gippini,}

Dr. en Ciencias Químicas

El nexo de unión de todos los capitulos del libro es la idea subyacente de crear una teoria general de pastas. Moldeo y Cocción son los dos procesos los que debe adecuarse la composición. Las caracteristicas fisico-quimicas más importantes que deben presentar las pastas para que los resultados de estos procesos sean satisfactorios y cómo pueden cambiarse dichas caracteristicas son los temas de discusión escogidos.

Un volumen encuadernado en cartoné, de $25 \times 17 \mathrm{~cm}$ compuesto de 259 páginas, 143 figuras y fotografias, y 37 tablas

Precios: 2.000 ptas.; \$ USA 29.00

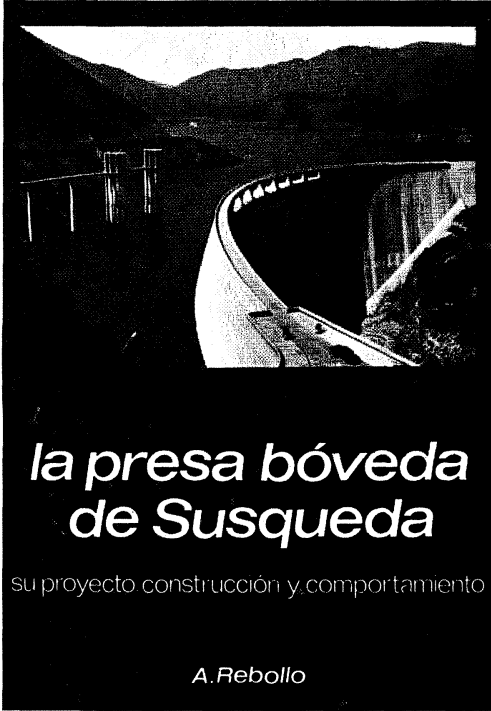

La presa bóveda de Susqueda

A. Rebollo,

Dr. Ingeniero de Caminos

El esfuerzo del constructor de presas se sitúa por su pretensión de perennidad, a contracorriente de las tendencias de la civilización actual, caracterizada por to fungible. Pueden evocarse las 10.000 grandes presas en funcionamiento o en construcción que están envejeciendo y reclaman los cuidados gerontológicos para mantener y perfeccionar su servicio y garantizar su inalienable pretensión de perennidad. En la medida en que todas nuevas obras, grandes o pequeñas, son portadoras de riesgos ecológicos y, a veces, catastróficos, que aumentan con el envejecimiento, la gerontologia de las presas es todo un emplazo. La acción adelantada de Arturo Rebollo en este terreno marca un camino a seguir para todos los que aman su propia obra con la devoción paternal que él ha puesto en Susqueda.

Un volumen encuadernado en cartoné plastificado con lomo de tela, de $18 \times 24,5 \mathrm{~cm}$, compuesto de 408 páginas, 330 figuras y fotografias y 39 tablas. Precios: 1.700 ptas.; extranjero, \$ USA 24.00 .

Precios: 1.800 ptas.; \$ USA 26.00 